\title{
Low-cost, green synthesis of highly porous carbons derived from lotus root shell as superior performance electrode materials in supercapacitor
}

\author{
Xin Wang ${ }^{\mathrm{b}, \uparrow}$, Mengjiao Wang ${ }^{\mathrm{b}, \uparrow}$, Xuemei Zhang ${ }^{\mathrm{b}}$, Hejun $\mathrm{Li}^{\mathrm{a}}$, Xiaohui Guo ${ }^{\mathrm{a}, \mathrm{b}, *}$
}

\begin{abstract}
Facile Production of high quality activated carbons from biomass materials has greatly triggered much attention presently. In this paper, a series of interconnected porous carbon materials from lotus root shells biomass are prepared via simple pyrolysis and followed by a $\mathrm{KOH}$ activation process. The prepared carbons exhibit high specific surface areas of up to $2961 \mathrm{~m}^{2} / \mathrm{g}$ and large pore volume $\sim 1.47$ $\mathrm{cm}^{3} / \mathrm{g}$. In addition, the resultant porous carbons serve as electrode materials in supercapacitor exhibit high specific capacitance and outstanding recycling stability and high energy density. In particular, their specific capacitance retention was almost $100 \%$ after 10500 cycles at a current density of $2 \mathrm{~A} / \mathrm{g}$. Remarkabely, the impact of the tailored specific surface areas of various carbon samples on their capacitive performances is systematically investigated. Generally, it was believed that the highly-developed porosity features (including surface areas and pore volume and pore size-distributions), together with the good conductivity of activated carbon species, play a key role in effectively improving the storage energy performances of the porous carbon electrode materials in supercapacitor.
\end{abstract}

Key words: Carbon, Porous, Biomass, Surface area, Capacity 


\section{Introduction}

Electrical double layer capacitors (EDLCs) are one of the promising electrochemical energy storage devices with high power characteristics [1-3], which have been proved to be useful in consumer electronics, memory backup systems, uninterruptable power sources, and hybrid electric vehicles $[4,5]$. EDLCs with nanostructured activated carbon (AC) electrode materials from natural products have attracted considerable attention due to their scalability, low-cost, excellent cycle stability, and attractive overall performances [5-8]. As a result, much effort has been devoted to increase the specific capacitance and energy density of carbon-based EDLCs that make them more capable for the primary power sources [9-16].

Generally, the energy storage performance of EDLCs is associated with the adsorption of electrolyte ions on large specific surface area (SSA) of carbon based electrodes. As we know that, high specific surface areas and accessible pores adapted to electrolyte ions are highly desired for EDLCs [17-24]. As a result, various synthetic methods have been attempted to enhance the SSA and pore volume of the carbon materials. For example, literatures reported that utilizing specific template techniques can prepare highly porous carbons [25-27], which exhibited excellent specific capacitances and rate capability. Another promising way to produce porous carbon materials with tailoring pore structure is the carbide-derived carbon (CDC) technique [28], but, their surface areas and pore volumes of the resultant CDCs are lower than those of zeolite-templated carbons. In addition, selective etching of metals from metal carbides and the formation of carbide-derived carbons (CDC) only offer very narrow pore structure [29-30], furthermore, they always suffer from limited SSA of micropores and tortuous pore shape, which would greatly limit their potential applications [31].

Recently, hydrothermal carbonization (HTC) was considered as one of well-developed industrial 
techniques to produce porous carbon from natural products due to its large-scale synthesis, low-cost, low-temperature, and environmentally-friendly advantages [32-36]. To date, various natural products (c.a. fungi, corn grain, lignocellulosic materials, sunflower stem, hair, and starch) derived porous carbons have been prepared via HTC treatment process [37-43]. Results discovered that the carbon products yield is high, the resultant carbons display tailoring porosity and show great potential as electrode materials in EDLCs. Unfortunately, the serious disadvantages of carbons originated from natural biomasses lack sufficient uniformity and consistency in their porous properties, which could be prohibitive for practical applications of EDLCs [44,45].

It should be pointed out; the classical activated carbon synthesis route via pyrolysis of organic species followed by an activation process is likely to be considered a promising commercial solution [46]. Thus, developing novel methods for the low-cost synthesis of ACs with large pore volume and high SSA and well-controlled microstructure are crucial for satisfy commercial EDLCs applications [47-51]. Wherein, we employ facile pyrolysis followed by a chemical activation process to convert a bio-waste lotus root shell into highly-developed porous carbon materials. As we know that, there are abundant lotus root shell in China and other countries [52], but only small proportion of lotus seed embedded in louts root are used as the traditional Chinese medicine and food and tea [53], main proportion product lotus root shell becomes wastes of markets and factories, thereby resulting great resources waste and environmental pollution. As a consequence, it is of great importance for developing low-cost, facile and scalable synthesis of highly-porous carbons from lotus root shell bio-wastes currently.

In our study, a series of lotus root shell-derived carbon species with different SSA and multiple pore-size-distributions (PSD) can be achieved via simple pyrolysis treatment followed by a $\mathrm{KOH}$ activation process, detailed preparation process can be seen in Figure 1(a). The prepared carbons display

ultra-high specific surface area of $2961 \mathrm{~m}^{2} \mathrm{~g}$-1, and large pore volume of $1.47 \mathrm{~cm}^{3} / \mathrm{g}$, and high energy density and excellent recycling durability in EDCLs. More importantly, the specific porous carbon materials with high SSA from biomass would highlight their potential in such as energy storage, catalytic, and environmental engineering fields.

\section{Experimental}

\subsection{Synthesis of porous carbon materials from lotus root shell}


In a typical synthesis, first, the lotus root shell was immersed in $\mathrm{HCl}(1 \mathrm{M})$ solution for $5 \mathrm{~h}$ at room temperature, and were thoroughly washed with distilled water and ethyl alcohol by ultrasonication and dried at $80{ }^{\circ} \mathrm{C}$ oven, then, the cleaned lotus root shell was cut to small blocks, and then was put into the quartz furnace at $350{ }^{\circ} \mathrm{C}$ for $1 \mathrm{~h}$ at a heating rate of $5{ }^{\circ} \mathrm{C} / \mathrm{min}$ under Argon gas flow and obtain the pre-carbonized carbon intermediates. Next step, the pre-carbonized carbons were mixed with activated reagent $\mathrm{KOH}$ (mass ratios $R_{\mathrm{KOH} / \mathrm{carbon}}=1 / 3,1 / 4,1 / 5, \mathrm{w} / \mathrm{w}$ ), respectively, then heated to the target temperatures $\left(700,800\right.$, and $\left.900{ }^{\circ} \mathrm{C}\right)$ at a heating rate of $5{ }^{\circ} \mathrm{C} / \mathrm{min}$ in a horizontal furnace under Argon atmosphere and held for $2 \mathrm{~h}$. After cooling down to room temperature, the resulted products were then thoroughly washed with $10 \mathrm{wt} \% \mathrm{HCl}$ solution to remove the residual inorganic residuum, and then rinse with distilled water until $\mathrm{pH}$ reach $\sim 7$. Finally, the resulted products were dried in an $80{ }^{\circ} \mathrm{C}$ oven for $12 \mathrm{~h}$. The resultant porous carbon materials are denoted as C-T-R, where $\mathrm{T}$ indicates the activation temperature and $\mathrm{R}$ indicates the mass ratios between $\mathrm{KOH}$ and pre-carbonized carbon intermediates.

\subsection{Characterization}

Fourier Transform infrared spectroscopy (FTIR) were recorded with Bruker EQUINOX-55 infrared spectrophotometer on $\mathrm{KBr}$ pellet. The thermogravimetry analysis (TGA) was carried out on a Rigaku standard TG-DTA analyzer with a heating rate of $10{ }^{\circ} \mathrm{C} / \mathrm{min}$ under nitrogen atmosphere. The morphology of the samples was observed by scanning electron microscope (VEGA-3, Tescan) with operation voltage of $15 \mathrm{kV}$ that equipped with energy-dispersive spectroscopy (EDS). Samples morphology/structure was observed by a FEI Quanta-400 FEG scan electron microscope (FE-SEM) with accelerating voltage of $200 \mathrm{kV}$. The porous features of the prepared samples are determined by Brunaur-Emmett-Teller (BET) surface analyzer (ASAP-2020, Micromeritics, USA). Prior to the measurements, all samples were degassed at $573 \mathrm{~K}$ until a stable vacuum of ca. 5 mTorr was reached. The Raman spectra was taken via using a Raman spectrometer (Jobin Yvon Co., France) model HR800 employing a $10 \mathrm{~mW}$ helium/neon laser at $632.8 \mathrm{~nm}$. X-ray photoelectron spectroscopy (XPS) was conducted using Model VG ESCALAB apparatus with an Al Ka x-ray source to analyze the surface composition of the samples.

\subsection{Electrochemical measurements}

All electrochemical evaluation was carried out using an electrochemical working station (CHI660E, Shanghai, China) in a three-electrode cell system at room temperature. The working electrode is 
fabricated by mixing the as-prepared carbon samples $(8 \mathrm{mg})$ with carbon black (super-P-Li) and polyvinylidene difluoride (PVDF) at a weight ratio of $8: 1: 1$. After thorough mixing, the slurry is pressed onto a piece of Nickel foam and then dried at $60{ }^{\circ} \mathrm{C}$ in a vacuum oven overnight (The effective area of the electrode is around $1.5 \mathrm{~cm}^{2}$ ). All electrochemical measurements were carried out at room temperature in a three-electrode system consisting of the carbon based powder electrode as working electrode, a $\mathrm{Ag} / \mathrm{AgCl}$ electrode as reference electrode, and a platinum wire electrode as counter electrode, respectively. The used electrolyte was $2 \mathrm{M} \mathrm{KOH}$ solution. Cyclic voltammetry (CV) was conducted within a potential range from -1.2 to $-0.6 \mathrm{~V}$ versus $\mathrm{Ag} / \mathrm{AgCl}$ electrode at various scan rates.

\section{Results and discussion}

A kind of black powder was obtained via a simple pyrolysis treatment at $350{ }^{\circ} \mathrm{C}$ furnace for $3 \mathrm{~h}$ followed by a $\mathrm{KOH}$ activation process at $700{ }^{\circ} \mathrm{C}$ under Argon atmosphere, as shown in Figure 1(b). It was observed that the prepared powder sample displays multiple-layer flake-like morphology; further high-resolution TEM (HRTEM) observation can find that the flake-like structure exhibits worm-like porous feature, as shown in Figure 1(c), the pore size was measured to be approximately $2 \mathrm{~nm}$. Further increasing activation temperature to $800{ }^{\circ} \mathrm{C}$, then a class of plate-like sample was obtained (Figure 1d), in addition, it was observed that that the specific plate was composed of multiple-layered flake units. From HRTEM image, we can find that C-800-4 sample displays obvious interconnected pore feature, its mesopore size was ranged from 2 to $3 \mathrm{~nm}$, as shown in Figure 1(e). Similarly, the C-900-4 sample was obtained by $\mathrm{KOH}$ activation at $900{ }^{\circ} \mathrm{C}$ furnace for $2 \mathrm{~h}$, thus, C-900-4 sample displays sheet-like morphology that analogous to graphene as shown in Figure 1(f), HRTEM observation find that the sheet structure possess well-defined mesoporous feature (Figure 1g). Like this, other black samples formed at different conditions can be seen in supporting information in Figures S1-3. Wherein, all the prepared porous samples were assigned to pure carbon species according to the presence of two typically characteristic peaks of carbon at Raman spectra, as shown in Figures S2(e) and S7. In particular, energy disperse spectrum (EDS) testing confirms the presence of $\mathrm{C}$, $\mathrm{O}$, and small amount of $\mathrm{S}$ in the sample C-800-4, as shown in Figure S2(d).

In order to study structural features of the prepared carbons, Fourier transform infrared (FTIR) spectra were measured at room temperature (Figure S8a). It was found that broad and strong peak at around $3457 \mathrm{~cm}^{-1}$ produced from the $v(\mathrm{O}-\mathrm{H})$ stretching vibration mode suggests that $-\mathrm{OH}$ group is 


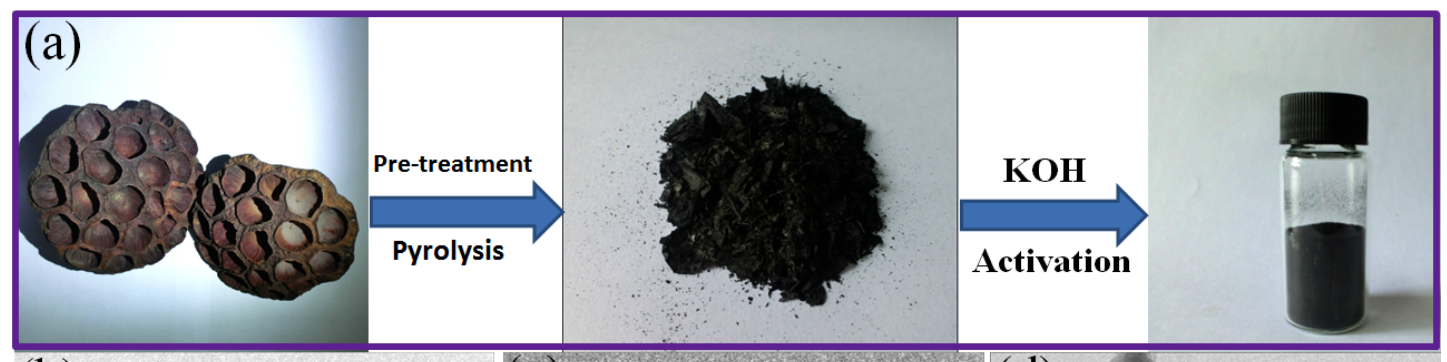

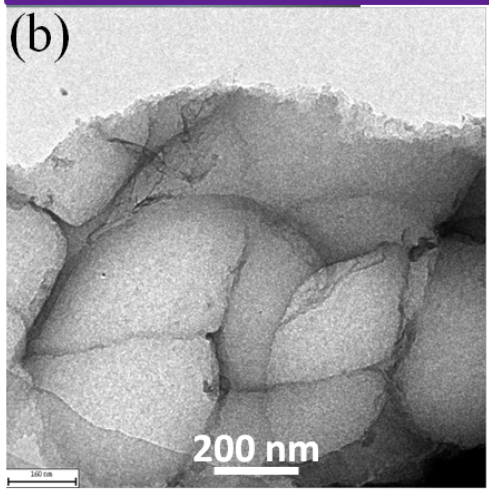

(c)
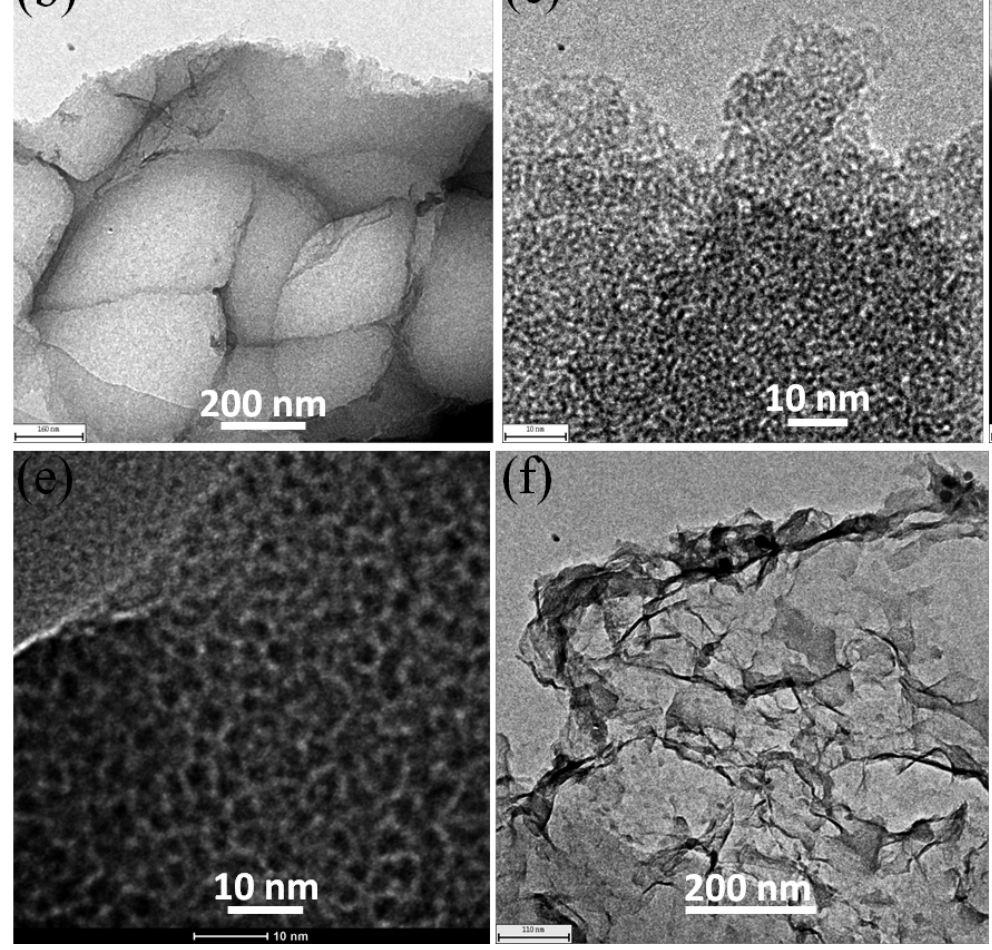

(d)
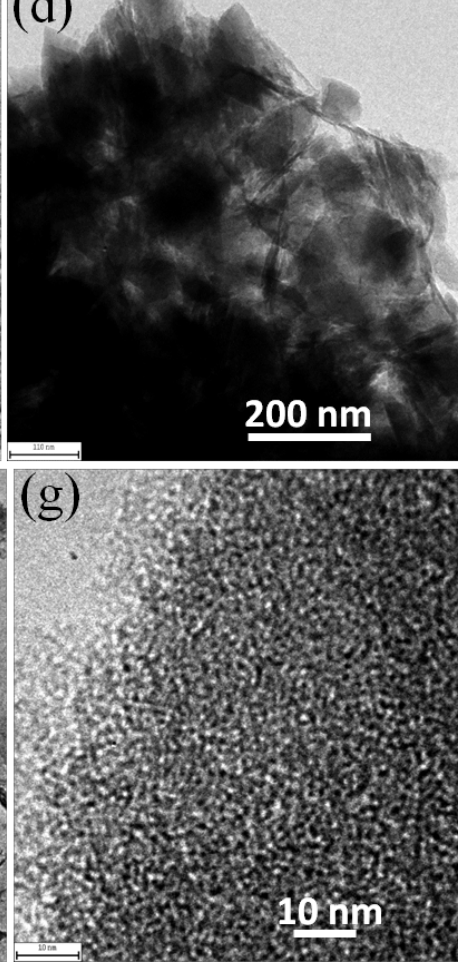

Figure 1. (a) the preparation process of porous activated carbon samples from lotus root shell; (b, c) TEM images of the prepared carbon C-700-4;

(d, e) TEM images of the prepared carbon C-800-4; (f, g) TEM images of the prepared carbon C-900-4.

partly deprotonated. In addition, the peak at $2928 \mathrm{~cm}^{-1}$ corresponds to the vibration mode of $-\mathrm{CH}_{2}$ group. The peak at $2362 \mathrm{~cm}^{-1}$ can be assigned to the vibration mode of $\mathrm{C}-\mathrm{C}$ bond. The characteristic peaks of $\mathrm{C}=\mathrm{O}$ group were centered at approximately 1672 and $1255 \mathrm{~cm}^{-1}$, respectively. The peak at $1504 \mathrm{~cm}^{-1}$ can be assigned to the stretching vibration mode of $\mathrm{C}=\mathrm{C}$ bond. The strong peak at approximately 1392 and $1098 \mathrm{~cm}^{-1}$ was assigned to the bending vibration modes of $-\mathrm{C}-\mathrm{O}-\mathrm{C}$ group. Additionally, the peak centered at $660 \mathrm{~cm}^{-1}$ was assigned to out-plane bending vibration of $-\mathrm{OH}$ group. These results demonstrate that the prepared carbons possess high oxygen content and therefore should be responsive to the $\mathrm{KOH}$ treatment and sequent activation [5], this case can be further evidenced by the XPS results in Figure S9.

After performing chemical activation with $\mathrm{KOH}$ for the obtained pre-carbonized intermediates, a class 
of porous carbon product was prepared. To further explore their porosity features, the isothermal $\mathrm{N}_{2}$ adsorption-desorption analysis was performed. The $\mathrm{N}_{2}$ adsorption-desorption isotherms at $77 \mathrm{~K}$ of the as-prepared carbons are plotted in Figures 2(a) and (b); other results were shown in Figures S10 and S11.wherein, the nitrogen adsorption isotherms display typical IV type curve. Additionally, judged form the loop nature of the nitrogen adsorption isotherm can suggest their specific micro-mesopore features. The specific surface area and pore volume information for the as-prepared carbon samples are summarized in Table 1. Remarkably, according to the corresponding Barrett-Joyner-Halenda (BJH) model curves, the prepared C-700-4, C-800-4, and C-900-4 samples display well-defined micro-mesopore size-distributions as shown in supporting information Figures S4-6.
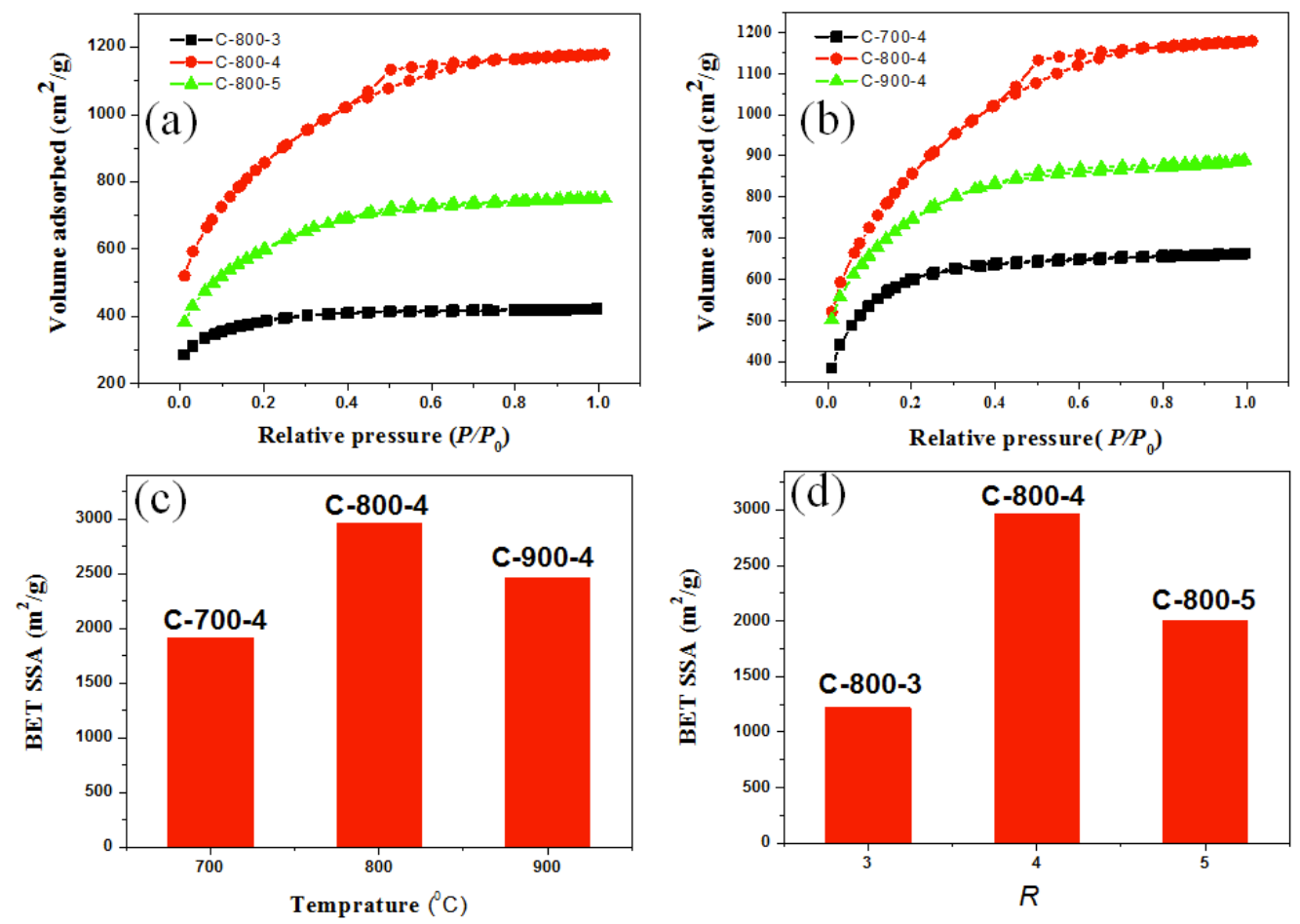

Figure 2. Nitrogen adsorption-desorption isotherms of carbon samples derived from lotus root shell at different reaction conditions; (a) various $\mathrm{R}$ values; (b) different activation temperatures in the case of $\mathrm{R} \sim 4$; (c) the relationship plots of activation temperatures and BET SSA; (d) the relationship plots of R and BET SSA.

It was known that $\mathrm{KOH}$ activation would generate micro/mesopores inside carbons, with the surface area and porosity being controlled by the activation temperature. As a result, the effect of activation temperatures on the SSA and PSD of carbons was explored via changing activation temperatures that ranged from 700 to $900{ }^{\circ} \mathrm{C}$ in the case of $R \sim 4$. Figure 2(c) presents the dependence of BET SSA on activation temperatures, in which, the SSA of the activated carbons was first increased 
from 1912 to $2961 \mathrm{~m}^{2} / \mathrm{g}$ and then rapidly decreased to $2464 \mathrm{~m}^{2} / \mathrm{g}$ with increasing activation temperature from 700 to $900{ }^{\circ} \mathrm{C}$, The best result came from the products under activation temperature of $800{ }^{\circ} \mathrm{C}$. This is due to the activity of $\mathrm{KOH}$ was highly increased from 700 to $800{ }^{\circ} \mathrm{C}$. Moreover, the effect of activation temperature on the PSD of the prepared carbons can be seen in Figures S4-6. Clearly, C-700-4, C-800-4, and C-900-4 exhibit highly-developed micropore features, pore-size-distribution was mainly ranged from 0.5 to $1.0 \mathrm{~nm}$.

\section{Table 1}

Enhanced summary of the porosity properties of the prepared carbon samples.

\begin{tabular}{llccccc}
\hline Entry & Samples & $\begin{array}{c}S_{\text {BET }} \\
\left(\mathrm{m}^{2} / \mathrm{g}\right)\end{array}$ & $\begin{array}{c}S_{\text {micro }} \\
\left(\mathrm{m}^{2} / \mathrm{g}\right)\end{array}$ & $\begin{array}{c}S_{\text {external }} \\
\left(\mathrm{m}^{2} / \mathrm{g}\right)\end{array}$ & $\begin{array}{c}V_{\text {pore }} \\
\left(\mathrm{cm}^{3} / \mathrm{g}\right)\end{array}$ & $\begin{array}{c}V_{\text {micro }} \\
\left(\mathrm{cm}^{3} / \mathrm{g}\right)\end{array}$ \\
\hline 1 & $\mathrm{C}-700-3$ & 1418 & 606 & 542 & 0.15 & 0.27 \\
2 & $\mathrm{C}-700-4$ & 1912 & 548 & 1364 & 0.38 & 0.17 \\
3 & $\mathrm{C}-700-5$ & 1,332 & 508 & 824 & 0.32 & 0.17 \\
4 & $\mathrm{C}-800-3$ & 1221 & 597 & 624 & 0.21 & 0.25 \\
5 & $\mathrm{C}-800-4$ & 2961 & 1132 & 1829 & 1.47 & 0.21 \\
6 & $\mathrm{C}-800-5$ & 2006 & 774 & 1234 & 0.76 & 0.20 \\
7 & $\mathrm{C}-900-3$ & 1214 & 592 & 622 & 0.14 & 0.27 \\
8 & $\mathrm{C}-900-4$ & 2464 & 1055 & 1409 & 0.77 & 0.33 \\
9 & $\mathrm{C}-900-5$ & 1751 & 826 & 925 & 0.39 & 0.18 \\
\hline
\end{tabular}

* The numbers of such as 3, 4 and 5 in C-T-R indicate the mass ratio values between pre-carbonized carbon intermediates and $\mathrm{KOH}$, respectively. $S_{\mathrm{BET}}$ is the total specific surface area; $V_{\text {pore }}$ is the total pore volume for the prepared carbon samples.

Moreover, the controlling of SSA can be realized by modulating the R values. To our knowledge, for the activation agent $\mathrm{KOH}$ which gave the best BET SSA results among the common activation agents [54], the $\mathrm{R}$ value was changed from 3 to 5 in the case of activation temperature $\sim 800{ }^{\circ} \mathrm{C}$, thus, the corresponding BET SSA was first increased from 1221 to $2961 \mathrm{~m}^{2} / \mathrm{g}$ and then gradually decreased to $2006 \mathrm{~m}^{2} / \mathrm{g}$ (Figure $2 \mathrm{~d}$ ). The highest SSA is achieved in the case of $R \sim 4$. These results demonstrate that varying $R$ values and activation temperatures can effectively modulate the SSA and PSD of the resultant carbons. 

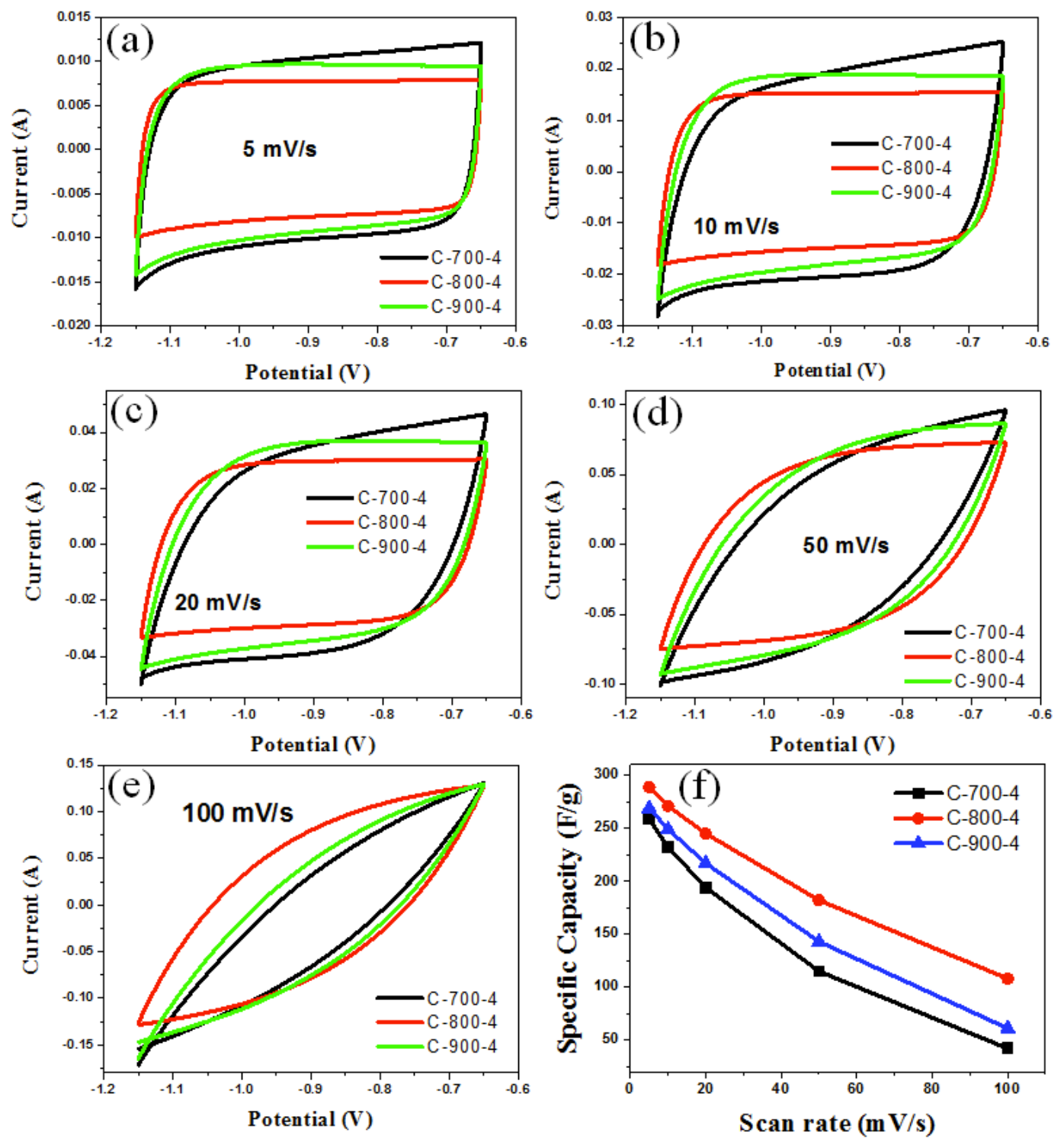

Figure 3. Cyclic voltammetry (CV) measurements of C-700-4, C-800-4, C-900-4 in $2 \mathrm{M} \mathrm{KOH}$ solution over a potential range from -1.2 to $-0.6 \mathrm{~V}$ at different scan rates (mV/s) of (a) 5, (b) 10, (c) 20, (d) 50 and (e) 100, respectively; (f) the correlation plots between scan rate and specific capacitances.

Additionally, the porous features of the electrode are critical to facilitate the mass transport of electrolytes within the electrodes for fast redox reactions and double-layer charging/discharging. Wherein, we investigate the electrochemical performances for the carbon-based electrodes in $2 \mathrm{M} \mathrm{KOH}$ solution. Their capacitive performances were measured via cyclic voltammetry (CV) technique. The typical CV curves for the obtained porous carbon electrodes in $2 \mathrm{M} \mathrm{KOH}$ electrolyte versus $\mathrm{SCE}$ at different scan rates of 5,10,20,50, and $100 \mathrm{mV} / \mathrm{s}$ as shown in Figure 3(a)-(e), then, the achieved specific capacitances are plotted in Figure 3(f). It was seen from Figure 3(f) that the specific capacitances for all carbon electrodes behave dramatically increase with the decrease of scan rates. In 
addition, the electrochemical data (CV and charge-discharge measurement) of the other carbons formed at different activated temperatures and R conditions were shown in Figures S12-14, herein, we find that C-900-5 carbon-based electrode displays high-rate feature compared with other carbon-based electrodes.
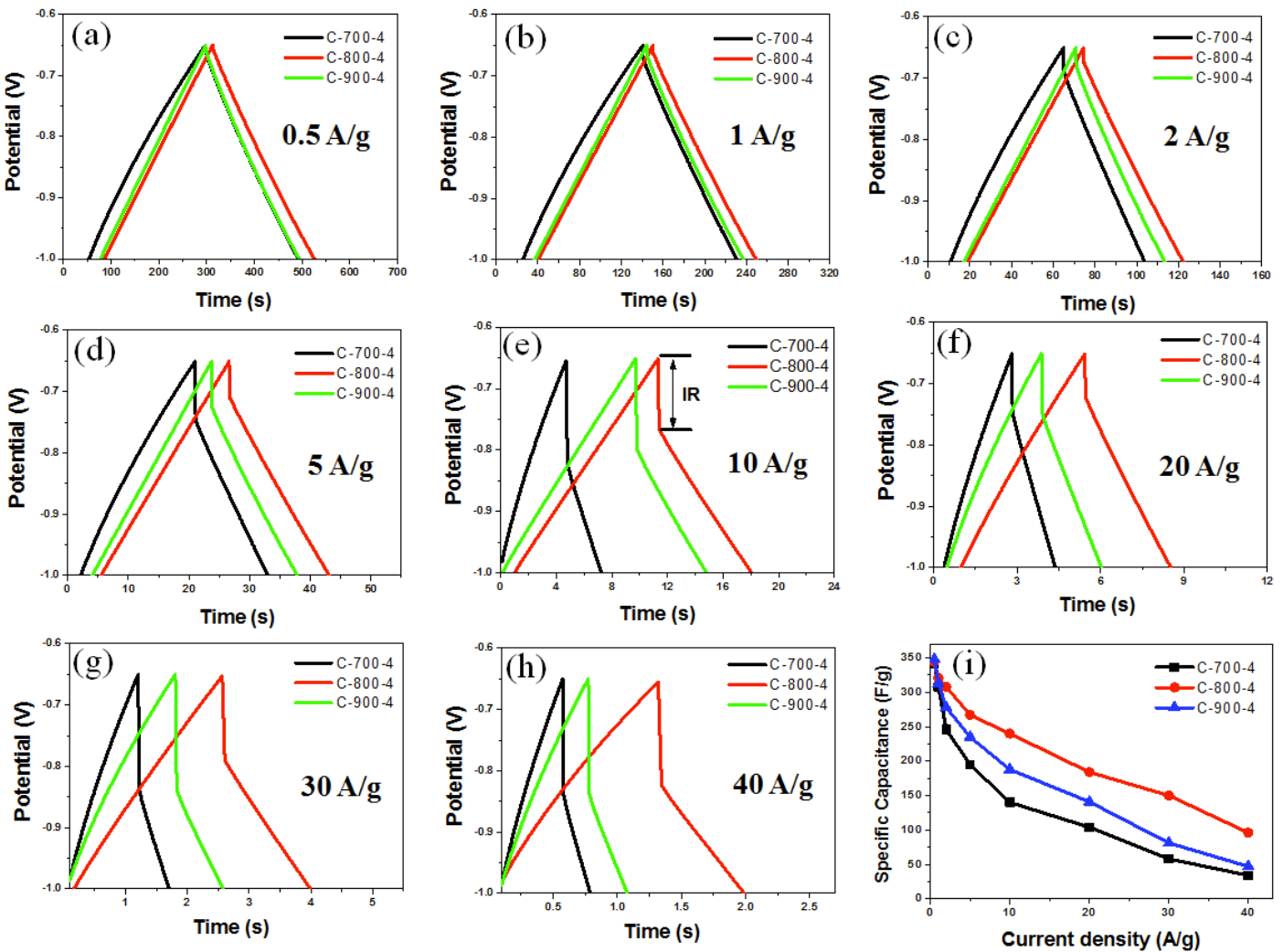

Figure 4. Charge-discharge curves of different carbons at different current densities. (a) 0.5, (b) 1.0, (c) 2.0, (d) 5.0, (e) 10, (f) 20, (g) 30, (h) 40, (i) the correlation curves between current density and specific capacitances.

In addition, galvanostatic charge-discharge measurements were conducted in $2 \mathrm{M} \mathrm{KOH}$ electrolyte between -1.2 and $-0.6 \mathrm{~V}$ (vs. SCE) at various current densities that ranged from 0.5 to $10 \mathrm{~A} / \mathrm{g}$, as shown in Figure 4. In which, the present distorted and/or quasi-linear shapes imply the occurrence of graphitization and improvement in conductivities [55] for C-700-4, C-800-4, and C-900-4 samples, respectively, as shown in Figure 4(a)-(h), wherein, the obtained symmetrical triangles of the charge/discharge plots at high current densities display obvious IR drop (especially in Figures 4e and h), Indicating an ideal EDLC behavior of the porous carbon electrodes [56]. In addition, Figure 4(i) presents the relationships between the specific capacitances and charge/discharge current densities. When the current densities were ranged from 0.5 to $40 \mathrm{~A} / \mathrm{g}$, the prepared carbon electrodes display tailoring capacitive performances. For example, at a current density of $0.5 \mathrm{~A} / \mathrm{g}$, the achieved specific capacitances of C-700-4, C-800-4, and C-900-4 are 340, 345, and $348 \mathrm{~F} / \mathrm{g}$, respectively, this case is related to the high 
accessible surface areas of the resultant carbons. These values are significantly higher than that of commercial activated carbons (270 F/g), when applied current densities of $0.5,1,2,5,10,20,30$ and 40 $\mathrm{A} / \mathrm{g}$, thus, the measured specific capacitances are plotted in Figure 4(i).
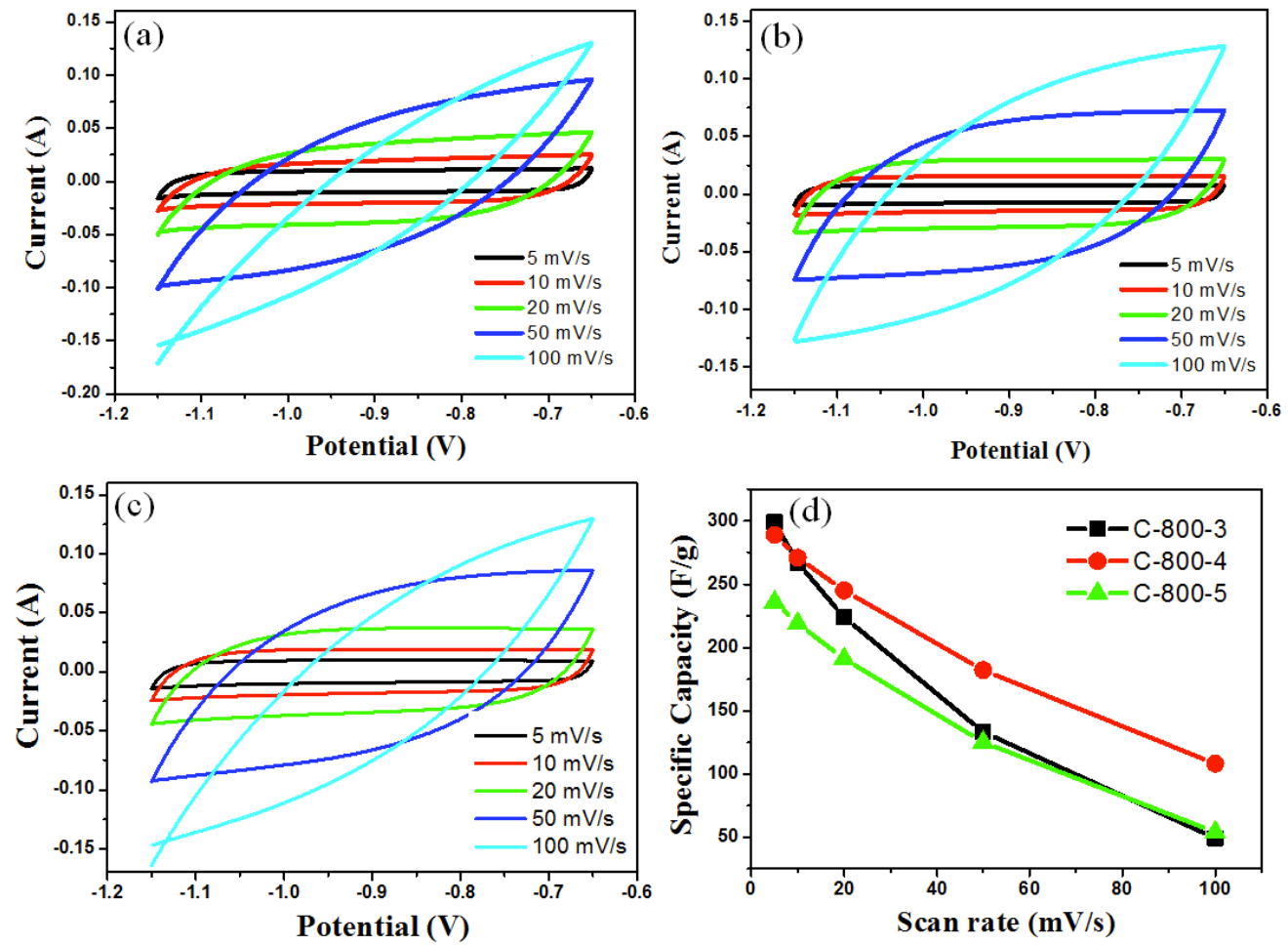

Figure 5. Cyclic voltammetry $(\mathrm{CV})$ measurements of the prepared carbons in $2 \mathrm{M} \mathrm{KOH}$ solution over a potential range from -1.2 to $-0.6 \mathrm{~V}$ at different scan rates. (a) C-800-3, (b) C-800-4, (c) C-800-5, (d) the correlation curves between scan rate and specific capacitances.

In particular, the specific capacitances of carbon C-800-4 electrode in two-electrode cell system are 205,188 and $179 \mathrm{~F} / \mathrm{g}$ for the current densities of $0.57,1.13$, and $2.83 \mathrm{~A} / \mathrm{g}$, respectively, these data are better compared with previously reported result of the carbon-based electrode [55-58], as shown in Figure S16. Noted that, the specific capacitances occur obviously decrease at high current densities, which is probably due to an inadequate time for electrolyte diffusion into the inner pores and the increment of interfacial polarity. It should be pointed out; the mesopores formed in the carbon materials are vital for furnishing a smooth and convenient charge transfer pathway and thus improve electrolyte accessibility to the microporous area. In which, C-800-4 possess much amounts of mesopores compared with C-700-4 and C-900-4 carbons, as clearly confirmed in Figures S4-6. 

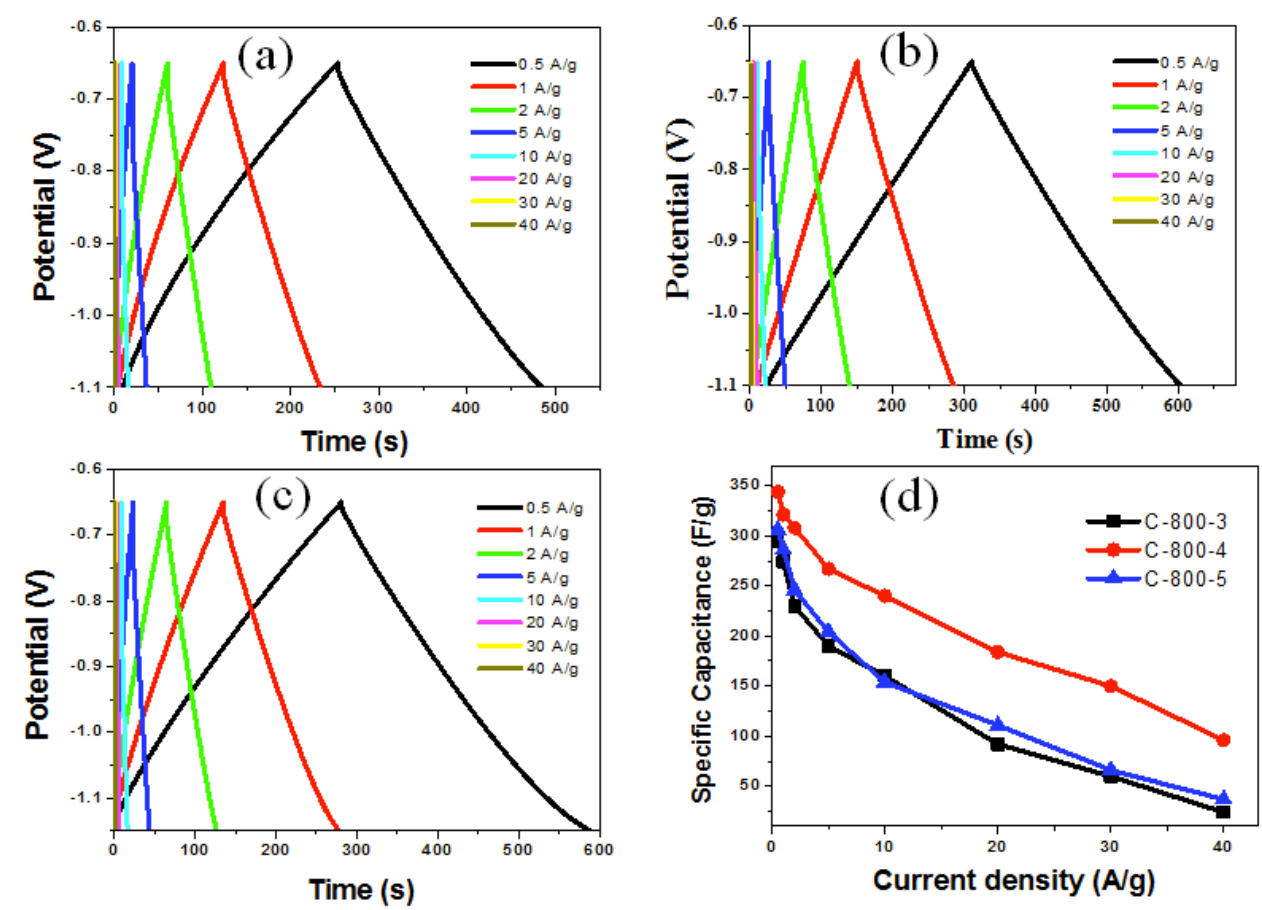

Figure 6. Charge-discharge curves of different carbons at different current densities. (a) C-800-3, (b) C-800-4, (c) C-800-5, (d) the correlation curves between current density and specific capacitances.

On the other hand, to further reflect the role of different $R$ values on the electrochemical performances of the porous carbons. The corresponding electrochemical Performances of the prepared carbon based electrodes were measured via CV testing technique. The typical CV curves for C-800-3, C-800-4, and C-800-5 electrodes in 2 M KOH electrolyte versus SCE are shown in Figure 5(a)-5(c), herein, some distorted rectangular shape for $\mathrm{CV}$ curves can be observed at higher scan rates, the distorted rectangular curves maybe ascribed with the relatively slow redox reaction kinetics due to the presence of oxygen-including functional groups in the carbons, This case can be evidenced in previous literature [43]. Additionally, the achieved specific capacitances are seen in Figure 5(d), it was found from Figure 5(d) that the specific capacitances for the three carbon electrodes behave dramatically increase with the decrease of the scan rates. Clearly, the mean decrease tendency of specific capacitance for C-800-4 behaves relatively smooth with the increment of scan rates, indicating fast capacitive response, which maybe related with the effective SSA in carbons [13].

Furthermore, for the prepared C-800- $R$ series samples at different $R$ values, their galvanostatic charge-discharge measurements were also conducted in $2 \mathrm{M} \mathrm{KOH}$ aqueous electrolyte (vs. SCE) at various current densities ranged from 0.5 to $10 \mathrm{~A} / \mathrm{g}$, as shown in Figure 6 . Wherein, the presented quasi-linear shapes imply the occurrence of graphitization and improvement in conductivities for 
C-800-3, C-800-4, and C-800-5 samples, as shown in Figure 6(a)-(c). In addition, Figure 6(d) indicates the relationships between the current density and specific capacitances. While applied current densities are ranged from 0.5 to $40 \mathrm{~A} / \mathrm{g}$, it was clearly seen that the prepared C-800-4 electrode displays the maximum capacitive performances with respect to the C-800-3 and C-800-5. For example, at a current density of $0.5 \mathrm{~A} / \mathrm{g}$, the calculated specific capacitances of C-800-3, C-800-4, and C-800-5 are 293, 345, and $306 \mathrm{~F} / \mathrm{g}$, respectively, indicating quick ion transport and high charge Storage in pores of carbons. As mentioned in Table 1, the calculated SSA for C-800-3, C-800-4, and C-800-5 are 1221, 2961, and 2006 $\mathrm{m}^{2} / \mathrm{g}$, respectively, higher SSA can provide a possibility for highly efficient penetration of electrolytes into the surface of active materials, thus increasing the utilization of active materials, additionally, the pore volume of C-800-4 is the highest than the C-800-3 and C-800-5, the data demonstrate that C-800-4 possess better charge-storage capability compared with C-800-3 and C-800-5, as a consequence, both high SSA and pore volume are facilitated to the enhancement of the collective capacitive behavior, which is well agreed with previously reported study $[13,58,59]$.

Meanwhile, to validate their potential application in EDLCs, the cyclic stability of the porous carbon C-800-4 electrode was examined by means of charge/discharge cycle testing, as shown in Figure 7(a). It was found that the specific capacitance retention was almost $100 \%$ after 10500 cycles at a current density of $2 \mathrm{~A} / \mathrm{g}$, implying the excellent recycling durability, which is superior to most previously reported carbon based electrodes $[58,60,61]$. Herein, the optimal cycling durability is likely due to the improved charge storage in pores of carbon or perhaps to in situ activation of the electrode to expose additional surface areas, herein, the C-800-4 possess high SSA $\sim 2961 \mathrm{~m}^{2} / \mathrm{g}$ and pore volume $\sim 1.47 \mathrm{~cm}^{3} / \mathrm{g}$, these data are critical to enhance the cycling stability. In addition, the presence of much more amounts of oxygen-containing functionalities in C-800-4 can also increase the accessible electroactive surface area, which can favor the enhancement of cycling stability. More importantly, the highly-developed micropores of C-800-4 (c.a. $0.5,0.6,0.75,1.05,1.08$, and $1.20 \mathrm{~nm}$ in micropore-size-distributions) would be well matched with the absorption of the aqueous electrolyte molecules in pores of carbons, which can indeed enhance recycling durability. 

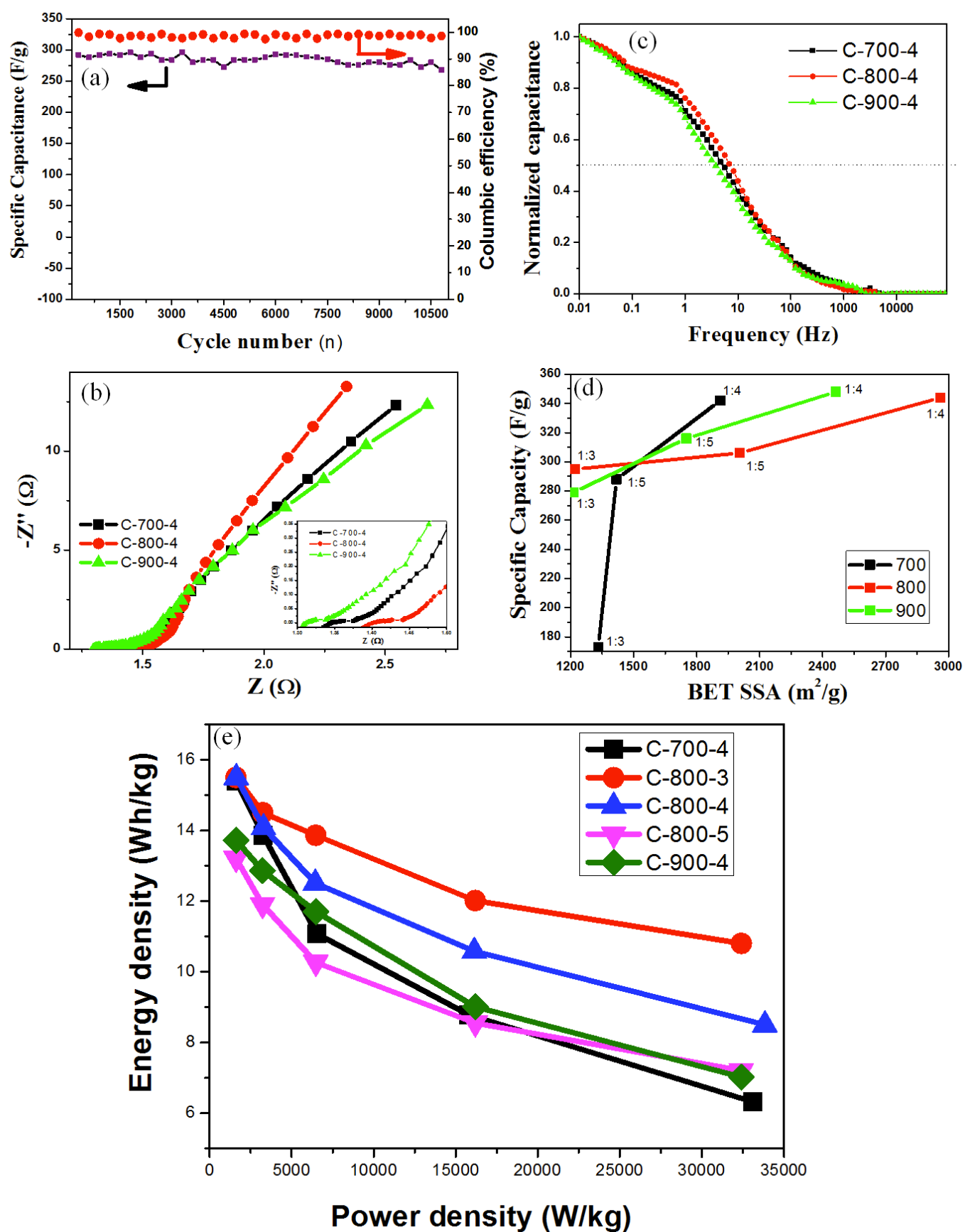

Figure 7. The recycling testing curve for the prepared C-800-4 sample at constant current density of 2 A/g (a); Nyquist Plots (b); frequency response curves (c); the correlation curves between BET SSA at different $R$ and specific capacitances (d); Relationship curves of power density and energy density for the prepared carbons (e).

To further understand their capacitive behaviors, we carried out electrochemical impedance spectroscopy (EIS) testing (Figure 7b). The resulting Nyquist plots exhibit semicircles in high frequency region, reflecting the existence of Rct (charge-transfer resistance). Wherein, the magnified high frequency region shows major differences. The bigger semicircle indicates a lower ionic conductivity of 
the electrolyte, and the relatively longer length of $45^{\circ}$ phase shift segment implies higher Warburg impedance [55]. The equivalent series resistances are measured to be $1.37,1.39$, and 1.38 for C-700-4, C-800-4, and C-900-4, respectively, showing good conductivity in aqueous electrolyte. Electrochemical impedance spectroscopy (EIS) provides complementary information about the frequency response of carbons in EDLCs (Figure 7c). The projected length of the Warburg-type line is related to the ion diffusion limitations within the electrode material [56]. Noted that, the operating frequency at which the capacitance is $50 \%$ of its maximum value was increased from 3.7 (C-700-4) to $5.6(\mathrm{C}-900-4)$ and $6.8 \mathrm{~Hz}$ (C-800-4), indicating the specific capacitance of C-800-4 exhibits fast frequency response compared with other two carbon based electrodes. These values of operating frequency are quite high; which are comparable to, even higher what was obtained for state-of-the-art ordered mesoporous carbide derived carbons $(0.1-0.7 \mathrm{~Hz}$ in an ionic liquid) and previously reported for optimized activated hydrothermal carbons $(0.1 \mathrm{~Hz}$ in organic electrolyte) and advanced polypyrrole-derived activated carbons $(\sim 0.06 \mathrm{~Hz}$ in ionic liquid electrolyte) and biomass derived carbons $(\sim 0.62 \mathrm{~Hz})[31,43,45,55]$.

In order to verify the role of the specific surface area in improving the electrochemical performances of the porous carbons, the relationship between SSA and specific capacitances were conducted, as shown in Figure 7(d), it was found that the three carbon samples display the minimum specific capacitances in the case of $R \sim 3$, their achieved specific capacitances behave the maximum values in the case of $R \sim 4$, this case strongly demonstrate that the increase of SSA indeed cause the enhancement of the specific capacitances, which is well coincided with previously reported results[13,45]. Remarkably, it was observed that the specific capacitances of C-800-R samples exhibit relatively stable tendency with respect to the other two kinds of carbon samples (C-700-R and C-900-R). Additionally, the Ragone plot for the porous Carbons based EDCLs was conducted, as shown in Figure 7(e), which reflects the relationships between energy density and power density. Thus, C-700-4, C-800-3, C-800-4, C-800-5, and C-900-4 exhibit high energy densities of $\sim 11.1,13.9,12.6,10.3$, and $11.7 \mathrm{Wh} / \mathrm{kg}$ at a power of $6.4 \mathrm{~kW} / \mathrm{kg}$, respectively. Wherein, it was found that the C-800-3 displays optimal gravimetric energy/power density, this is due to the smallest electrode density compared with other carbons. This data full demonstrates that the prepared porous carbon based electrodes would highlight their great potential in EDLCs industry.

Taken together, the superior capacitive performances of C-T-R based supercapacitors could be 
attributed to the following aspects: 1) highly interconnected porous channels including well-defined micro-mesopores can ensure significantly improve the accessibility of the electrolyte ions to active materials, and greatly shorten the diffusion and migration paths of electrolyte ions during the rapid charge/discharge process; 2) high SSA, large pore volume, and adjustable PSD with short pore length of C-T-R are favorable for forming a larger amount of double electric layers and facilitates the transport of the electrolyte ions and accumulating much charge/ions in pores of carbons; 3) the presence of oxygen-containing-functionalities in carbons maybe improve the accessible electroactive surface area as well as provide additional pesodocapacitance. The synergic effects from above three aspects would effectively enhance the collective capacitive performances of the prepared carbon based electrodes in EDLCs.

\section{Conclusions}

We first have demonstrated that a class of highly porous activated carbons was successfully prepared via simple pyrolysis followed by a chemical activation process. Herein, the prepared C-800-4 sample displays ultra-high specific surface area $\sim 2961 \mathrm{~m}^{2} / \mathrm{g}$ and large pore volume $\sim 1.47 \mathrm{~cm}^{3} / \mathrm{g}$ and plentiful PSD features. More important, the resultant porous carbons as electrode materials in EDLCs exhibit high specific capacitance and outstanding recycling stability. Furthermore, benefiting from the specific carbons with ultra-high SSA and large pore volume and tailoring PSD, low-cost, scalable, and green synthetic approach would open the possibility to commercial storage energy devices and could be extended to the applications as adsorbents, supporter for catalysis, environmental engineering and biomedical-related materials.

\section{Acknowledgments}

We acknowledge the National Science Foundation of China (No. 21173167), the Science and Technology Committee of Shaanxi Province (Grant No. 2014KW09-03), Program for New Century Excellent Talents in University (NCET-13-0953), and the Research Fund of the State Key Lab of Solidification Processing (NWPU), China (Grant No 15-BZ-2015). We specially thank help from Prof. Yanwu Zhu in providing two-electrode testing and HRTEM testing.

\section{References}


[1] Hall P J, Mirzaeian M, Fletcher S I, Sillars F B, Rennie J R, Shitta-Bey G O. Energy Environ Sci, 2010, 3: 1238

[2] Lee S W, Gallant B M, Byon H R, Hammond P T, Yang S H. Energy Environ Sci, 2011, 4: 1972

[3] Lota G, Fic K, Frackowiak E. Energy Environ Sci, 2011, 4: 1592

[4] Simon P, Gogotsi Y. Nat Mater, 2008, 7: 845

[5] Miller J R, Simon P. Science, 2008, 32: 651

[6] Lin Y H, Wei T Y, Chien H C, Lu S Y. Adv Energy Mater, 2011, 1: 901

[7] Yuan C Z, Zhang X G, Su L H, Gao B, Shen L F. J Mater Chem, 2009, 19: 5772

[8] a) Wang B, Zhu T, Wu H B, Xu R, Chen J S, Lou X W. Nanoscale, 2012, 4: 2145; b) Li Z H, Wu D

C, Huang X, Ma J H, Liu H, Liang Y R, Fu R W, Matyjaszewski K, Energy Environ Sci, 2014, 7:3006

[9] Yuan C Z, Xiong S L, Zhang X G, Shen L F, Zhang F, Gao B. Nano Res, 2009, 2: 722

[10] Frackowiak E. Phys Chem Chem Phys, 2007, 9: 1774

[11] Wang Y, Shi Z, Huang Y, Ma Y, Wang C, Chen M. J Phys Chem C, 2009, 113: 13103

[12] Gogotsi Y, Simon P. Science, 2011, 334: 917

[13] Zhang L, Yang X, Zhang F, Long G K, Leng K, Huang Y. J Am Chem Soc, 2013, 135: 5921

[14] An G H, Ahn H J. Carbon, 2013, 65: 87

[15] Simon P, Gogotsi Y. Acc Chem Res, 2012, 46: 1094

[16] Ghosh A, Lee Y H. Chemsuschem, 2012, 5: 480

[17] Jha N, Ramesh P, Bekyarova E, Itkis M E, Haddon R C. Adv Energy Mater, 2012, 2: 438

[18] Bichat M P, Raymundo-pinero E, Beguin F. Carbon, 2010, 48: 4351

[19] Wei T Y, Chen C H, Chien H C, Lu S Y, Hu C. Adv Mater, 2010, 22: 347

[20] Arico A S, Bruce P, Scrosati B, Tarascon J M, Van-Schalkwijk W. Nat Mater, 2005, 4: 366

[21] Mini P A, Balakrishnan A, Nair S V, Subramanian K R V. Chem Commun, 2011, 47: 5753

[22] Meng F, Fang Z, Li Z, Xu W, Wang M, Liu Y, J Mater Chem A, 2013, 1: 7235

[23] Xu F, Tang Z W, Huang S Q, Chen L, Liang Y R, Mai W C, Zhong H, Fu R W, Wu D C, Nat Commun, 2015, 6:7221

[24] Liang Y R, Fu R W, Wu D C, ACS NANO, 2013, 7:1748

[25] Li Z H, Wu D C, Liang Y R, Fu R W, Matyjaszewski K, J Am Chem Soc, 2014, 136:4805

[26] Kyotani T, Ma Z X, Tomita A. Carbon, 2003, 41: 1451

[27] Wu D C, Li Z H, Zhong M J, Kowalewski T, Matyjaszewski K, Angew Chem Int Ed, 2014, 53:3957 
[28] Chmiola J, Yushin G, Gogotsi Y, Portet C, Simon P, Taberna P L. Science, 2006, 313: 1760

[29] Yushin G, Dash R K, Gogotsi Y, Jagiello J, Fischer J E. Adv Funct Mater, 2006, 16: 2288

[30] Korenblit Y, Rose M, Kockrick E, Borchardt L, Kvit A, Kaskel S, Yushin G. ACS NANO, 2010, 4: 1337

[31] Rose M, Korenblit Y, Kockrick E, Borchardt L, Oschatz M, Kaskel S. Small, 2011, 7: 1108

[32] Hu B, Wang K, Wu L, Yu S H, Antonietti M, Titirici M M. Adv Mater, 2010, 22: 813

[33] Titirici M M, Antonietti M. Chem Soc Rev, 2010, 39: 103116

[34] Sevilla M, Fuertes A B. Chem Eur J, 2009, 15: 4195

[35] Sevilla M, Fuertes A B. Carbon, 2009, 47: 2281

[36] Sevilla M, Fuertes A B, Mokaya R. Energy Environ Sci, 2011, 4: 1400

[37] Zhao L, Fan L Z, Zhou M Q, Guan H, Qiao S. Adv Mater, 2010, 22: 5202

[38] Zhu H, Wang X L, Yang F, Yang X R. Adv Mater, 2011, 23: 2745

[39] Balathanigaimani M S, Shim W G, Lee M, Kim C, Lee J W, Moon H. Electrochem Commun, 2008, 10: 868

[40] Wu F C, Tseng R L, Hu C, Wang C. J Power Sources, 2004, 138: 351

[41] Chen W X, Zhang H, Huang Y Q, Wang W. J Mater Chem, 2010, 20: 4773

[42] Qian W, Sun F, Xu Y, Qiu L, Liu C, Wang S, Yan F. Energy Environ Sci, 2014, 7: 379

[43] Wei L, Sevilla M, Fuertesm A B, Mokaya R, Yushin G. Adv Energy Mater, 2011, 1: 356

[44] Kim P, Joo J B, Kim W, Kim J, Song I K, Yi J. Catal Lett, 2006, 112: 213

[45] Lu W, Marta S, Fnertes A B, Mokaya R, Yushin G. Adv Funct Mater, 2012, 22: 827

[46] Frackowiak E, Beguin F. Carbon, 2001, 39: 937

[47] Basta A H, Fierro V, El-Saied H, Celzard A. Bioresour Technol, 2009, 100: 3941

[48] a) Lu W, Yushin G. Nano Energy, 2012, 1: 552; b) Liang Y R, Wu D C, Fu R W, Sci Rep, 2013, $3: 1119$

[49] Li Z, Xu Z, Tan X, Wang H, Holt C M B, Stephenson T. Energy Environ Sci, 2013, 6: 871

[50] Yun Y S, Cho S Y, Shim J, Kim B H, Chang S J, Baek S J, Adv Mater, 2013, 25: 1993

[51] Biswal M, Banerjee A, Deo M, Ogale S. Energy Environ Sci, 2013, 6: 1249

[52] Akiyaama K, Matsuzaki K, Hayashi H. Nature, 2005, 435: 824

[53] Zeng H Y, Cai L H, Cai X L, Wang Y J, Li Y, Q J. Sci Food Agr, 2013, 5: 1070

[54] Murali S, Potts J R, Park J, Stoller M D, Zhang L, Ruoff R S. Carbon, 2012, 50: 3482 
[55] Wang H, Xu Z, Kohandehghan A, Li Z, Cui K, Holt C M B. ACS NANO, 2013, 7: 5131

[56] Wu X L, Wen T, Guo H L, Yang S, Wang X, Xu A W. ACS NANO, 2013, 7: 3589

[57] Zhu Y, Murali S, Stoller M D, Ganesh K J, Cai W, Ferreira P J. Science, 2011, 332: 1537

[58] Jin Z Y, Lu A H, Xu Y, Zhang J T, Li W C. Adv Mater, 2014, 26, 3700-3705

[59] Yoon Y, Lee K, Baik C, Yoo H, Min M, Park Y. Adv Mater, 2013, 5: 4437

[60] Yan J, Liu J P, Fan Z J, Wei T, Zhang L J. Carbon, 2012, 50: 2179

[61] Subramanian V, Luo C, Stephan A M, Nahm K S, Thomas S, Wei B Q. J Phys Chem C, 2007, 111: 